\title{
Structuring Measurements for Modeling and the Deployment of Industrial Wireless Networks
}

\author{
Rong Zhang ${ }^{1}$, Zeljko Zilic ${ }^{1}$ and Katarzyna Radecka ${ }^{2}$ \\ ${ }^{1}$ Department of ECE \\ McGill University \\ Montreal, QC, Canada H3A $2 A 7$ \\ \{zhangr,zeljko\}@macs.ece.mcgill.ca \\ ${ }^{2}$ Department of ECE \\ Concordia University \\ Montreal, QC, Canada H3G 1 M8 \\ kasiar@ece.concordia.ca
}

\begin{abstract}
The deployment of wireless networks in the industrial settings is hampered by the inability to predict the wireless link performance in the field. We present the methodology for extracting models and assessing the performance in the deployment that is based on the packet reception rate. The measurements taken with IEEE 802.15.4-compliant wireless networks extract the relationship between the location (distance) and communication properties (packet reception rate) under different wireless network feature configurations (such as output power level, packet size, asymmetry, channels, temporal, antenna features etc.) We then outline means to build usable models of wireless networks and to evaluate and tune the performance of deployed wireless networks.
\end{abstract}

\section{Introduction}

The true convenience of interconnecting devices without the use of wires has lead to the unprecedented success of wireless technologies in the computer and consumer electronics industry [8]. Various wireless networks are now beginning to appear in industrial settings. They promise to reduce the cost and save the time needed for the installation and maintenance of industrial control networks. The number of cables normally required in such an environment can be substantially reduced, thus making plant setup and reconfiguration easier. For example, a typical commercial building can contain hundreds of sensors that are wired to central air conditioning and ventilation systems. Replacing wired units with wireless sensor network (WSN) nodes offers more flexibility, and ultimately a better and more efficient installation. Eliminating wiring is especially important in industrial environments where chemicals, vibrations, or moving parts can damage any cabling.

Wireless networking technology poses, however, many challenges [9]-[11], especially in guaranteeing the sufficient wireless coverage during its deployment. Wireless networking devices are inherently power-limited, which limits the ability to combat communication channel errors. Even without power limitations, phenomena such as obstruction and multipath interference on the transmitted signal path make the link quality hard to predict and design for. The industrial setting, with numerous pieces of metal machinery, racks and moving parts is especially plagued by link obstruction and multipath interference. If a transmitter node (TX) is trying to connect to a receiver (RX) located in a typical industry environment full of metal surfaces (as shown in Figure 1), there will be many transmission paths, including a direct Line-of-sight (LOS) connection path and other multiple-reflection Non-line-of-sight (NLOS) paths. Since each path has different delay and attenuation, the received signal is badly affected by those destructive interferences.

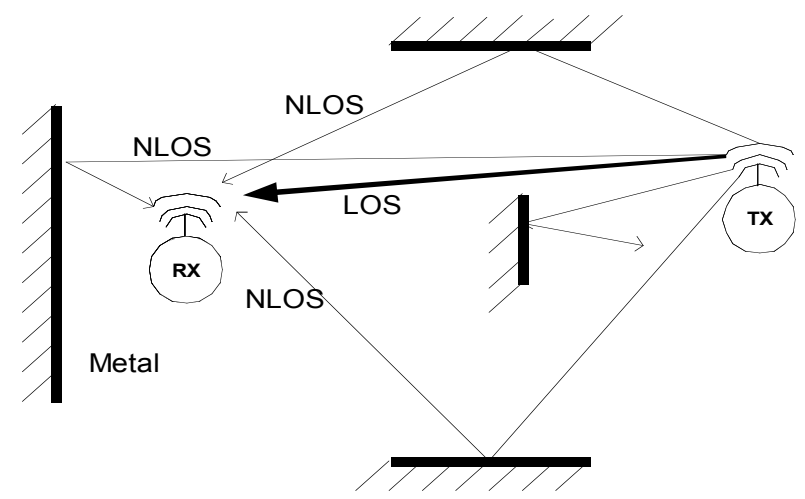

Figure 1: Wireless Links in an Industrial Setting

The performance of deployed wireless networks greatly depends on the details of the underlying communication channel [4]. Hence, to evaluate performance of wireless networks, an accurate communication model is necessary. Until recently, two major approaches have been in widespread use in the sensor network community: unit disk modeling and empirical data traces [4]. The unit disc model states that communication between two wireless nodes is solely a function of the distance and that communication is conducted without any loss of packets if the nodes are closer than a specified communication range. However, the complete correlation between the properties of geometric space and the topology of the network has been refuted by numerous experiments in actual deployments [1]-[3]. At the other end of the spectrum is the use of empirical traces of deployed systems. While these are completely accurate samples of real wireless networks, it is difficult to create from traces alone a large number of network instances that are properly characterized [4].

Recently, a statistical model of lossy links for wireless 
sensor network (WSN) was proposed [4] to produce network models of arbitrary sizes with realistic properties. This work provides a foundation for extracting the relationship between wireless node locations (distance) and the reception rate (RR) using non-parametric statistical techniques. The objective is to use a non-parametric method to obtain a Probability Density Function (PDF) that completely characterizes the relationship between the distance and RR. Based on the study of PDF about properties of individual and group links, an iterative improvement-based optimization procedure is used to generate network instances that are statistically similar to empirically observed network.

The IEEE 802.15.4 standard [5] was finalized in October 2003 with the aim of creating a low cost, low power, two-way wireless communication solution that meets the requirements of sensors and control devices. In contrast to other wireless protocols such as IEEE 802.11, IEEE 802.15.4 has been specifically developed for use with devices that require relatively low bandwidth to transmit data packets. Such applications exactly match the needs of many industrial environments. The unique properties of wireless links in the 2.4-GHz range (commonly used in IEEE 802.15.4) are: radio waves can penetrate walls and are reflected by several materials. Errors occur not only due to noise, but also due to the multipath fading. In addition, there is the distance-dependent path loss and co-/adjacent channel interference on the channel. Hence, the wave propagation environment (number of propagation paths, their respective loss) and its time-varying nature (moving people, machines or wireless nodes) play a decisive role.

In this paper, we present a methodology for conducting measurements for the purpose of assisting in their seamless deployment. We apply this methodology to the IEEE 802.15.4 wireless protocol. The chosen measurement scenario shares some common characteristics of industry environments: many metal surfaces, moving parts, and machines switching on and off. A targeted set of measurements about the physical and communication properties of WSN are presented. The measurement results can be used to set up experimental models for WSN [4] or to evaluate the performance of wireless networks prior to their deployment to a particular site [7]. Based on the measurement results, we provide the foundation for analyzing the influence of these features to the WSN performance and validate their suitability for the actual deployment.

\section{Measurement Methodology}

It is beneficial to parameterize the current wireless link models from "real data", obtained from measurements, or to use the measurement results as a motivation for developing better models. For the deployment of wireless networks, some network features measurements in real application environment can be used to evaluate the performance of targeted wireless networks and give a guideline for the deployment. The measurement setup is described next.

To investigate the wireless communication characteristics, we develop a series of measurements to build the statistical relationships with respect to the features that impact network architecture and protocols in real networks. Our task is to analyze the relationship between two main properties of wireless network under different working conditions. The investigated test features are shown in Table 1.

Table 1: Wireless Network Testing Features

\begin{tabular}{|c|c|}
\hline $\begin{array}{c}\text { Testing } \\
\text { features (Mi) }\end{array}$ & Testing configuration (Ci) \\
\hline $\begin{array}{c}\text { Transmit } \\
\text { Power level }\end{array}$ & $\begin{array}{c}\text { 8 output power levels ranging from } 0 \mathrm{dBm} \\
\text { to }-25 \mathrm{dBm}\end{array}$ \\
\hline Frequency & $\begin{array}{c}\text { Ranges from } 2.405 \mathrm{GHz} \text { to } 2.48 \mathrm{GHz}, 16 \\
\text { channels in total, monitors frequency } \\
\text { interference in different channels }\end{array}$ \\
\hline Packet size & 20,50 and 100 bytes per packet \\
\hline $\begin{array}{c}\text { Antenna } \\
\text { polarization }\end{array}$ & $\begin{array}{c}0,45,90 \text { degree difference between } \\
\text { transmitter and receiver antenna }\end{array}$ \\
\hline $\begin{array}{c}\text { Antenna } \\
\text { height }\end{array}$ & $0,50 \mathrm{~cm}$ height to the ground \\
\hline $\begin{array}{c}\text { Asymmetry } \\
\text { Temporal }\end{array}$ & $\begin{array}{c}\text { Detect the difference of transmission } \\
\text { direction of A->B and B- }>\text { A }\end{array}$ \\
\hline $\begin{array}{c}\text { Monitor the relationship of RR and } \\
\text { distance during different time }\end{array}$ \\
\hline
\end{tabular}

Transmit power levels: One of the fundamental issues that arise naturally in sensor network is the coverage. In radio communications, coverage means the geographical area within which service from a radio communications facility can be received. Energy is another key concern with wireless networks. The power level measurements consider the coverage and power issues at the same time.

Frequency: It is becoming commonplace to use the same unregulated ISM frequency band. For the protocols at the same band, it is necessary to investigate the performance of coexisting networks and to find methods for reducing mutual disturbance between them. The associated interference between IEEE 802.15.4 and IEEE 802.11 is quantitatively assessed here.

Packet size: The network performance measurements with different packet sizes are used to quantitively assess the influence of some protocol design to the wireless communication performance.

Antenna features: The proper location of WSN node and the orientation with respect to the antenna directionality can help to reach better coverage, as well as reduce the power consumption of node.

Asymmetry and Time-variable characteristics: The measurements presented here try to answer the following 
questions: Is there an asymmetry in WSN links? Does the temporal variability cause the change of wireless communication?

The above measurements are implemented by a pair of WSN nodes, as shown in Figure 2.

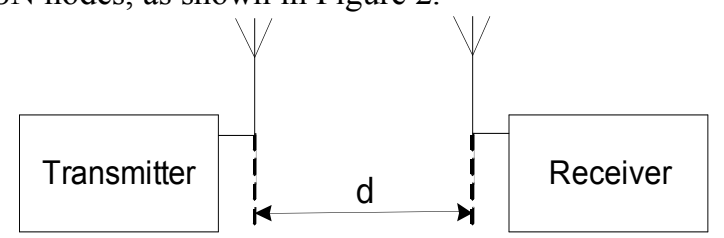

Figure 2: Wireless Network Measurement Architecture

The pseudo-code for transmitter and receiver are shown as follows:

Transmitter:

(For each test feature Mi) \{

Set the default configuration of TX node;

(For each test configuration $\mathrm{Ci}$ ) \{

Send the new configuration to RX node;

Change the TX node with new config;

If ( $T X$ and RX node are working in new configuration)

\{Sending packets ;\}

Receive test result ;

Set back to the default config ;\}

\section{Receiver:}

(For each test features Mi) \{

Set the default configuration of RX node;

(For each test configuration $\mathrm{Ci}$ ) \{

Receive new configuration from TX node;

Change the RX node with new config;

Counting the packets received;

Send the test result to TX node ;\}

Set back to the default configuration ;\}

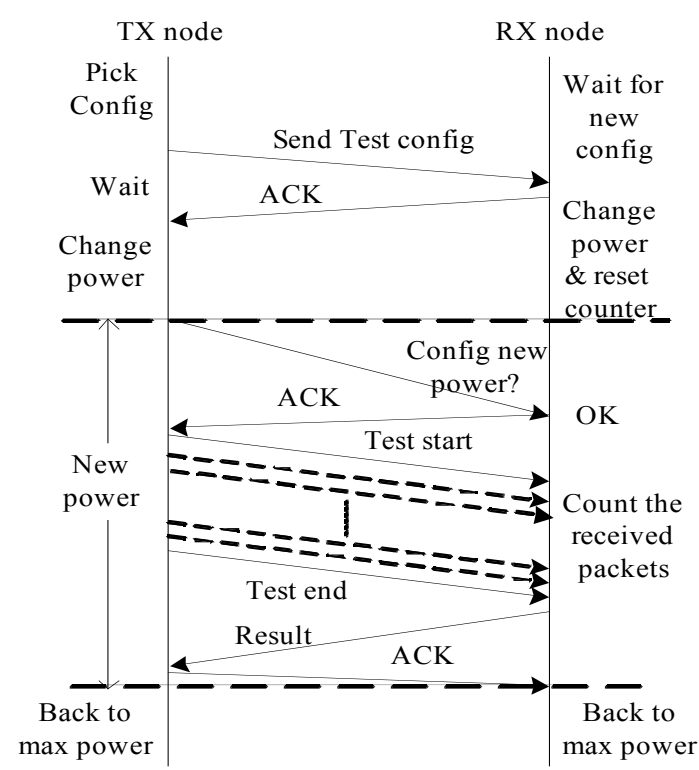

Figure 3: Wireless Network Testing Sequence Chart
Figure 3 shows the sequence chart of the code to test the relationship among RR, distance and output power levels. The testing code for other features has a similar program sequence.

\section{Understanding Measurement Results}

Our testing is implemented in two kinds of environments: the laboratory (indoors) and the campus (outdoors). The typical indoor environment includes the furniture (mental or wood), walls, electronic equipments, e.g. printers, microwave oven. The WSN node is built with the low power microcontroller MSP430 from TI, a Zigbee compliant RF transceiver CC2420 from Chipcon (delete [12]) and our own printed antenna [12]. A pair of nodes with the same hardware is placed at the increasing distances with or without line-of-sight between them. The outdoor environment includes trees and buildings. The pair of nodes are placed at the varying distances and (delete at) heights, such as near the ground or elevated off the ground. The indoors and outdoors testing environments are zones covered by the McGill wireless signal (802.11.b). At each test position, 10,000 test packets are transmitted for RR (delete packet reception rate) testing. In summary, the data set used in our testing consisted of packet delivery data for more than 2 million packets in experiments performed in 2 different environments, 8 different output power settings, 3 different working channels, 3 different packet sizes, 3 kinds of antenna polarization, 2 different antenna heights, two transmission directions for asymmetry and 10 different time points.

\subsection{Power levels}

Power level is an important characteristic of a wireless network node for power optimisation techniques. Here, the measurements are used to consider the tradeoffs between the coverage and energy consumption. For the employed Chipcon CC2420 RF transceiver [5], there are 8 programmable output power levels in total. Table 2 shows the relationship between output power and current consumption under different power levels. We test the relationship between RR and distance under each output power level.

Table 2: Output power under different power levels

\begin{tabular}{|c|c|c|}
\hline Power level & $\begin{array}{c}\text { Output Power } \\
(\mathrm{dBm})\end{array}$ & $\begin{array}{c}\text { Current Consumption } \\
(\mathrm{mA})\end{array}$ \\
\hline 8 & 0 & 17.4 \\
\hline 7 & -1 & 16.5 \\
\hline 6 & -3 & 15.2 \\
\hline 5 & -5 & 13.9 \\
\hline 4 & -7 & 12.5 \\
\hline 3 & -10 & 11.2 \\
\hline 2 & -15 & 9.9 \\
\hline 1 & -25 & 8.5 \\
\hline
\end{tabular}

Figure 4 shows the 3-D graph of the measured 
relationship between distances, power level and RR in outdoor environment. From this graph we can see that for a fixed power level, RR decreases as the distance is increased. For a fixed distance, RR decreases as the power level is decreased. At the highest output power level, the communication range can reach to $60 \mathrm{~m}$ with the required packet $R R$. This testing can be used to optimize the power consumption of the wireless node. With compliance to the required RR and distance, the output power of the CC2420 should be set as low as possible.

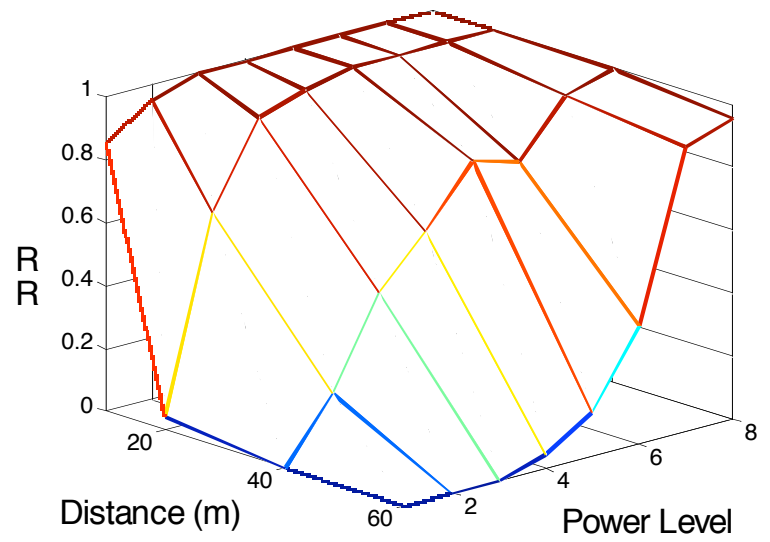

Figure 4: Reception vs. Power Level/Distance

\subsection{Asymmetry}

Asymmetry in communication refers to the difference in RR of packets communicated strictly between two nodes. Two cases are possible for nodes A and B- first, the transmitter is $\mathrm{A}$ and the receiver is $\mathrm{B}$; second, the transmitter is $\mathrm{B}$ and receiver is $\mathrm{A}$. When the difference is beyond $50 \%$, asymmetry is considered to be happening [4]. Very often, it is assumed that RR is the same in both directions. We design the tests to capture whether there is an asymmetry in the RR (as a function of the node distance).

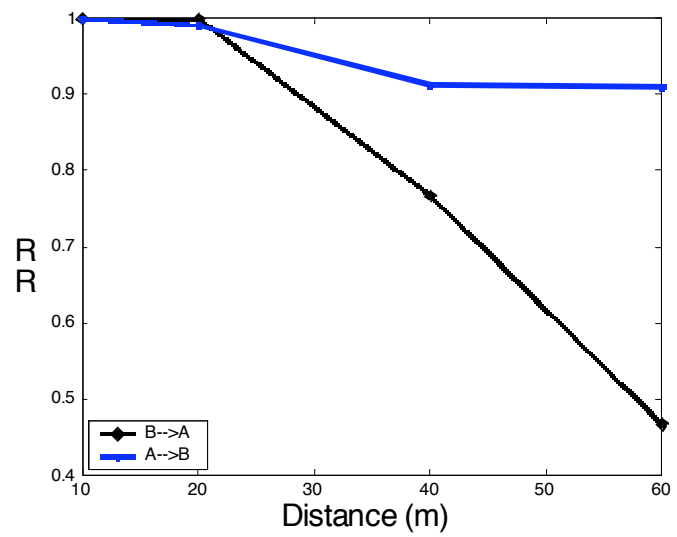

Figure 5: Asymmetric Reception Rates

Figure 5 shows the dependency of asymmetric RR as a function of distance in outdoors environment. From the result shown in Figure 5, we can see there is big difference $(44 \%)$ when the distance is very long $(60 \mathrm{~m})$.
By the definition of asymmetry (50\% difference), it may not be consider as asymmetry. The possible reason for the big difference could be the minor circuit differences between $\mathrm{A}$ and $\mathrm{B}$.

\subsection{Temporal Variability}

The goal of this measurement is trying to find the influence of the measurement time to the communication link performance (RR). Figure 6 shows the temporal variability of the relationship between RR and distance in indoor environment. The test is taken from 11AM to 9PM at two hours intervals. There is no obvious trend shown in the graph. There is only a minor difference (all from 97\% to $100 \%$ ) in the RR. The possible reason is the interference (e.g. machinery, microwave ovens, etc.) applied randomly over time. We observe that time is not an important factor influencing the relationship between RR and distance.

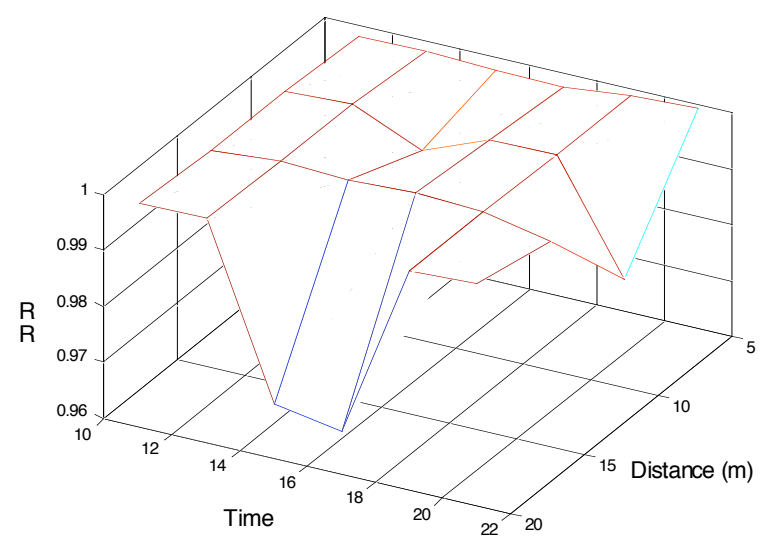

Figure 6: Time Dependency in Reception Rate

\subsection{Interference from other wireless networks}

Multiple wireless standards can use the same frequency band. The $2.4 \mathrm{GHz}$ ISM band is used for both IEEE 802.15.4 and IEEE 802.11 standards. The next measurement is designed to give the quantitive assessment about the interference between different wireless networks.

For the 802.15.4 networks [5], there are totally 16 channels (channel 11 to channel 26), located between 2.405 and $2.48 \mathrm{GHz}$, with channel spacing of $5 \mathrm{MHz}$. The measurement tries to capture the influence of different frequency to communication performance. Since the printed antenna is narrowband and designed to tune to Channel $11(2.405 \mathrm{GHz})$, channel 11 is expect to be the strongest channel with the highest RR compare to other channels. Figure 7 shows the relationship between RR and distance for three different channels (channel 11, 26 and 17) indoors. 


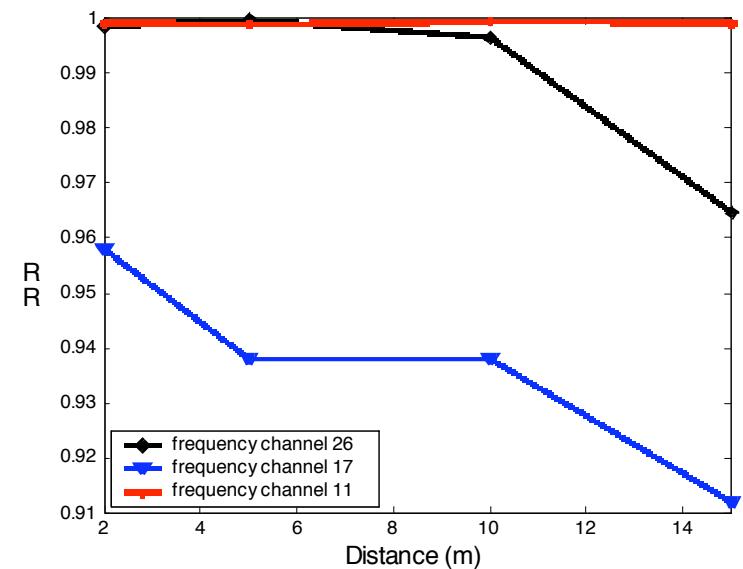

Figure 7: Dependency on Channels

From the measurement result, we can see the RR of channel 17 is much lower than channel 11 and channel 26. The RR of channel 11 is better than channel 26 . The reasons for these observations are explained as following:

1. Using a spectrum analyzer, we can see that there is a wide range of interference within the frequency band ranges from $2.43 \mathrm{GHz}$ to $2.45 \mathrm{GHz}$. The strongest interference is caused by the 802.11 b wireless internet access (both indoor and outdoor environments).

2. The printed antenna is tuned to channel 11; therefore the antenna performance is best for this channel. That explains that the RR in channel 11 is higher than $R R$ in channel 26 at each distance, even though there is no wireless interference in both channel 26 and channel 11.

\subsection{Packet size}

This measurement is used to detect the dependency of the transmission packet size to the RR and distance. Figure 8 shows the relationship between RR and distance for three packet sizes ( 20 bytes, 50 bytes and 100 bytes) in an indoor environment.

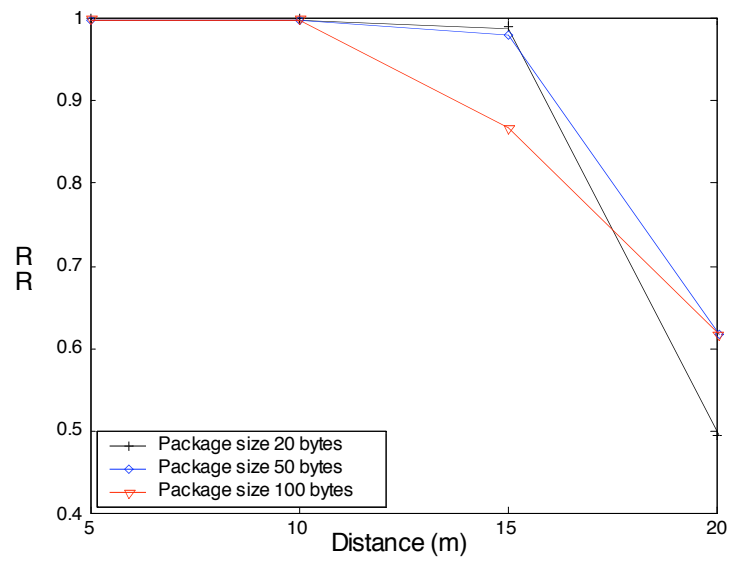

Figure 8: Packet Size Influence on RR
From Figure 8, we can see that when the distance is short, the minimum packets size ( 20 bytes) corresponds to the best RR. When the distance is long, that tendency is not present. From the test result, there is no obvious regularity for different packet sizes. We can conclude that packet size is not one of the important factors affecting communication properties.

\subsection{Antenna Features}

Antenna is a critical component of wireless node and its design plays an important role for the whole wireless network performance. Here, we present the measurement for two kinds of features of the antenna. One is the antenna polarization and the other is the antenna height above the ground. The measurement results can provide a guideline for refining the antenna design.

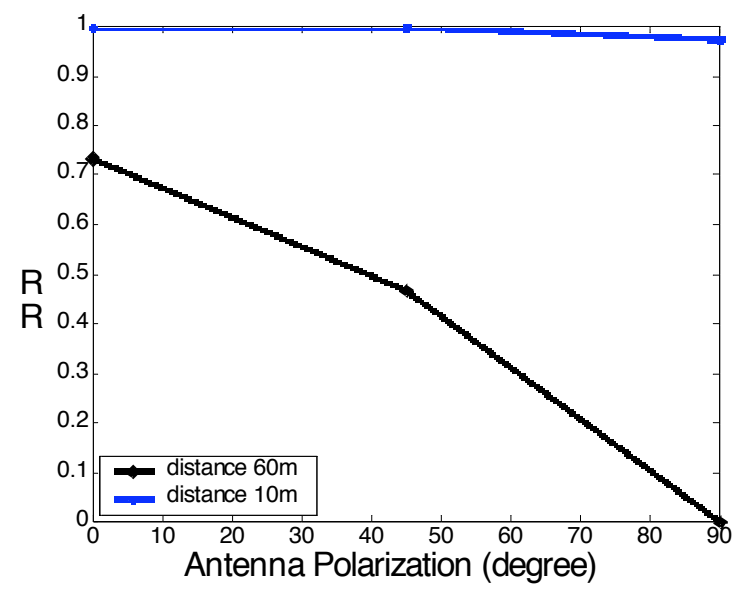

Figure 9: Dependency on Antenna Polarization

Figure 9 shows the dependency of RR and antenna polarization at two distances $(10 \mathrm{~m}$ and $60 \mathrm{~m})$ in outdoor environment. There are 3 antenna polarization tested in Figure 9: 0 degree (the antennas of transmitter and receiver are parallel), 45 degree ( 45 degree angle between antennas of transmitter and receiver) and 90 degree (the antennas of transmitter and receiver are perpendicular). From the results shown in Figure 9, the RR is highest when the antennas are parallel ( 0 degree) and the lowest RR happened when the antennas are vertical (90 degree). This result provides a guideline for the node location of wireless network. The antennas of the nodes in the real wireless network should be a parallel set. Also we can see from Figure 9, the effect of antenna polarization at short distance $(10 \mathrm{~m})$ is not as strong as at long distance $(60 \mathrm{~m})$. The possible reason is that when distance is long, the transmission signal is weak and easily affected by antenna polarization. 


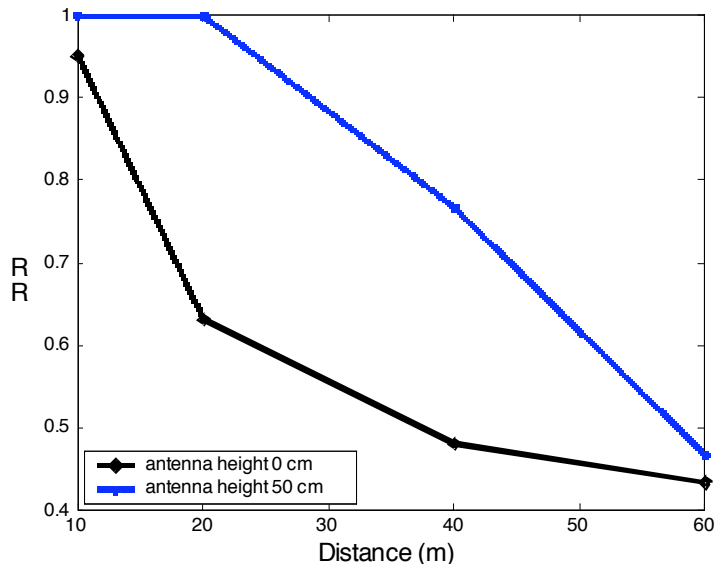

Figure 10: RR vs. Distance from the Ground

Figure 10 shows the relationship between the RR and distance at two antenna heights in outdoor environment. We set two test conditions: putting the wireless node on ground and at a height of $50 \mathrm{~cm}$ to ground. Based on the testing presented above, we can see that the antenna design and its placement are important factors for the system performance.

\subsection{Facilitating Deployment and Model Building}

We have presented a measurements setup that explores the relationship between distance and RR, asymmetry and temporal variation, etc. It can be used to build statistical models for WSN [4] in a straightforward way. The collected data can be the original data that the model needs to obtain the probability density function (PDF) that establishes a complete characterization of the relationship among various network features. The PDF provides the likelihood that any particular value of one feature is associated with a given value of another feature. Based on the PDF of realistic network features, a series of wireless network generators are developed to produce networks of an arbitrary size. The generated instances of the network are statistically similar to the empirically observed networks.

Coverage is a key parameter in evaluating the wireless networks prior to or upon their deployment in an industrial site. The coverage characterization can be easily obtained from our power levels measurement routines. Channels measurement shows the interference from coexisting wireless networks in the real industrial application. Both antenna polarization and antenna height shows that proper node location will increase the coverage as well as decrease the power consumption. Power and coverage are all key concerns to the deployment of wireless networks.

\section{Conclusions}

In this paper, we have developed a methodology for measuring a set of network features for characterizing links in wireless network communication. Our measurements can help building communication link models for an arbitrary network that is statistically similar to observed networks. These measurements also greatly impact the power management techniques and WSN node location and configuration. For example, the antenna polarization and height should be considered during node placement. With required packet RR and distance, the output power levels can be configured as low as possible to reduce the node energy consumption with required coverage. The insight gained while building these relationships gives a guideline for developers of protocols, localized algorithms and antenna design for wireless networks and the users of wireless products.

\section{Reference:}

[1] A. Cerpa, N. Busek and D. Estrin, "SCALE: A tool for simple connectivity assessment in lossy environments", Technical Report 0021, Center for Embedded Networked Sensing, UCLA, Sep 2003.

[2] D. Ganesan, B. Krishnamachari, A. Woo, D. Culler, D. Estrin and S. Wicker, "Complex behavior at scale: An experimental study of low power wireless sensor networks," Technical Report 02-0013, Center for Embedded Networked Sensing, UCLA, February 2002.

[3] Y. Zhao and R. Govindan, "Understanding packet delivery performance in dense wireless sensor networks," in Proceedings of ACM Sensys, Nov. 2003, pp. 1-13.

[4] A. Cerpa, J.L. Wong, L. Kuang, M. Potkonjak and D. Estrin, "Statistical Model of Lossy Links in Wireless Sensor Networks", in Proc. of Fourth International Symposium on Information Processing in Sensor Networks, April 2005, pp.81 88.

[5] IEEE std. 802.15.4/D18 - 2003: Wireless MAC And Physical Layer specifications for Low Rate Wireless Personal Area Networks.

[6] Chipcon AS SmartRF CC2420 Datasheet, 2004.

[7] P.S. Neelakanta and H. Dighe "Robust factory wireless communications: a performance appraisal of the Bluetooth ${ }^{\mathrm{TM}}$ and the ZigBee ${ }^{\mathrm{TM}}$ colocated on an industrial floor", in Proc. of the $29^{\text {th }}$ Annual Conference of the IEEE Industrial Electronics Society, Nov. 2003, pp. 2381-2386.

[8] A. Willig, D. Matheus and A. Wolisz, "Wireless Technologies in Industrial Networks", Proc. of the IEEE, June 2005, pp. 1130-1151.

[9] I. F. Akyildiz, W. Su, Y. Sankarasubramaniam, and E. Cayirci, "Wireless sensor networks: A survey," Computer Network. , pp. 393-422, 2002.

[10] H. Karl and A. Willig, Architectures and Protocols for Wireless Sensor Networks. Wiley, 2005.

[11] A. J. Goldsmith and S. B. Wicker, "Design challenges for energy-constrained ad hoc wireless networks," Wireless Comm., Aug. 2002. pp. 8-27.

[12] Milos Prokic, Jean-Samuel Chenard, Rong Zhang and Zeljko Zilic, "Methodology for rapid development and robust deployment of wireless sensor networks", Proc. Micronet Workshop, May 2005, pp. 103-104.

[13] M. Yan, A.P. Brown, R.A. Iltis, T. Sherwood, H. Lee and R. Kastner, "MP core: algorithm and design techniques for efficient channel estimation in wireless applications", Proc. Design Automation Conf. June 2005, pp. 297- 302. 